\title{
Indicadores de efetividade da vigilância epidemiológica para paralisias flácidas agudas no Brasil de 1990 a 2000
}

\author{
Eduardo Souza Teixeira-Rocha ${ }^{1}$ e José Tavares-Neto ${ }^{1}$
}

RESUMO Objetivos. Descrever e comparar os indicadores de qualidade de vigilância epidemiológica para paralisias flácidas agudas nos períodos pré (1990 a 1994) e pós-certificação (1995 a 2000) do Brasil como zona livre da poliomielite.

Métodos. Foram consideradas as seguintes variáveis: prevalência mínima (notificação $\geq 1$ caso em 100000 indivíduos com menos de 15 anos de idade), notificação negativa (ausência de casos informada semanalmente por no mínimo $80 \%$ das unidades notificadoras em cada região), tempo de investigação do caso (investigação de $80 \%$ ou mais dos casos notificados de paralisias flácidas agudas até 48 horas depois da notificação) e investigação diagnóstica (coleta de duas amostras de fezes para cultura viral nas 2 semanas seguintes ao início da deficiência motora em $80 \%$ ou mais dos casos de paralisias flácidas agudas). Os resultados foram classificados como adequados se fossem iguais ou superiores ao valor considerado como efetivo, ou como inadequados se estivessem abaixo desse valor. Foi calculada a razão percentual de valores adequados alcançados em cada unidade federada em cada ano, para cada período do estudo. Também foram apresentadas as médias finais por período, unidade e região.

Resultados. Considerando os resultados para o Brasil como um todo, o indicador de prevalência mínima apresentou redução de 1,3 para 0,9 por 100000 menores de 15 anos no período pós-certificação, mas a diferença não foi significativa $(\mathrm{P}>0,08)$. A notificação negativa manteve valores médios adequados nos dois períodos (87 e 84\%), mas a razão percentual de efetividade foi 6,6\% menor no período pós-certificação ( $\mathrm{P}>0,21)$. O tempo de investigação de casos de paralisia flácida aguda melhorou sua efetividade em 10,9\% (P > 0,06) no período póscertificação. A coleta de fezes permaneceu inadequada nos dois períodos (54 e 52\%).

Conclusões. Apesar da ausência de casos autóctones de poliomielite por vírus selvagem no Brasil no período pós-certificação, são ainda desfavoráveis os indicadores de qualidade da vigilância epidemiológica, o que pode retardar a detecção e investigação de casos de paralisias flácidas agudas e comprometer o programa de erradicação global da poliomielite. As novas estratégias de informação que estão sendo disponibilizadas para o sistema de saúde do Brasil poderão promover uma melhora na efetividade do programa nacional de controle da poliomielite.

Palavras-chave Poliomielite, vigilância epidemiológica, indicadores de qualidade em assistência à saúde, Brasil.

1 Universidade Federal da Bahia, Faculdade de Medicina, Salvador (BA), Brasil. Correspondência e pedidos de separatas devem ser enviados a Eduardo Souza Teixeira-Rocha no seguinte endereço: Rua Marechal Andréa 142, apto. 601, CEP 41820-090, Salvador, BA, Brasil. E-mail: duda.teixeira@ uol.com.br
Nas Américas, a eliminação do vírus selvagem da poliomielite, proposta pela Organização Mundial da Saúde (OMS) e Organização Pan-Americana da Saúde (OPAS) em 1985 (1), foi alcançada no Brasil em 1994 (2) — ou seja,
5 anos depois da ocorrência do último caso, em agosto de 1989, no Estado da Paraíba. Em 1988, devido ao progresso observado no continente americano, foi estabelecida pela OMS a meta da eliminação global da pólio para o ano 
2000, e criada a Iniciativa Global para Erradicação da Poliomielite (IGEP)(3).

No entanto, em 2000, a poliomielite por vírus selvagem ainda estava presente em cerca de 20 países do mundo, inclusive com regiões na África e Ásia consideradas reservatórios de poliovírus (4). As guerras, os baixos indicadores de desenvolvimento humano, a má estruturação dos serviços de saúde pública e a escassez de recursos foram as principais causas apontadas para 0 atraso no cronograma inicialmente proposto. Mesmo assim, entre 1988 e 2000, houve decréscimo de $95 \%$ no número de casos mundiais de poliomielite, sendo o número notificado em 1999 o menor da história da IGEP(5). No início de 2001, a OMS estabeleceu para o ano de 2005 a nova meta de erradicação (4), com base na expectativa de financiamento e na capacidade de o programa ser implantado dentro daquele momento histórico (6).

Conforme descrito nas estratégias para a erradicação da poliomielite quando do estabelecimento da primeira meta, para o ano de 2000, o conhecimento acerca da efetividade da vigilância epidemiológica das paralisias flácidas agudas (PFA) e do seu monitoramento é imprescindível para manter a eliminação e evitar a reintrodução do vírus selvagem (7). Considerando a experiência adquirida nas Américas, Pacífico Ocidental e Europa, as condições inadequadas de vigilância podem deixar sem percepção, até por mais de 3 anos, a transmissão de baixa intensidade do vírus selvagem da poliomielite (8).

No Brasil, o principal critério estabelecido pela comissão nacional de avaliação do programa de eliminação da poliomielite, em 1994, para estabelecer a erradicação da doença, foi a ausência de casos autóctones confirmados virologicamente durante pelo menos 3 anos na presença de vigilância adequada (2). Para serem considerados "adequados", os indicadores de efetividade da vigilância deveriam estar acima dos níveis sugeridos como ideais pela IGEP. A ausência de poliovírus detectáveis na comunidade, a avaliação dos processos de controle por especialistas do país e a adoção de me- didas apropriadas para lidar com os eventuais casos importados de zonas endêmicas foram os outros três critérios utilizados como parâmetro no processo de certificação do Brasil como zona livre de poliomielite por vírus selvagem.

O objetivo do presente estudo foi descrever e comparar os indicadores de qualidade de vigilância epidemiológica para PFA nos períodos pré (1990 a 1994) e pós-certificação (1995 a 2000) do Brasil como zona livre da poliomielite, de acordo com os critérios de efetividade da IGEP.

\section{MATERIAIS E MÉTODOS}

Foi realizado um estudo observacional descritivo de valores agregados, tipo ecológico, utilizando os dados de vigilância epidemiológica de PFA. Os dados do período pós-certificação foram obtidos junto à Fundação Nacional de Saúde (FUNASA) do Brasil e se referem ao período de 1995 a 2000, posterior à eliminação da poliomielite no Brasil. Os dados do período précertificação (1990 a 1994) foram extraídos do dossiê elaborado para a certificação do programa de eliminação da transmissão da poliomielite por vírus selvagem (2).

Para a montagem do banco de dados e configuração dos resultados foram consideradas as seguintes variáveis (9): prevalência mínima, notificação negativa, tempo de investigação do caso e investigação diagnóstica. Em termos de prevalência mínima, a taxa de notificação de casos de PFA deve ser de, no mínimo, um caso por 100000 habitantes menores de 15 anos de idade. Esse parâmetro corresponde à expectativa de casos da síndrome de Guillain-Barré (10) na faixa etária em questão, sendo o valor já ajustado para a América Latina (11). Se o sistema for sensível para detectar a prevalência de uma condição de manifestação clínica idêntica à poliomielite, como é o caso da síndrome de Guillain-Barré, a ausência de casos estará assegurada.

Quanto à notificação negativa, no mínimo $80 \%$ das unidades notificadoras de cada região devem informar se- manalmente a ausência de casos. Uma unidade de notificação é um hospital ou qualquer outro serviço de saúde que preste assistência direta à população da região de referência, com capacidade para detectar um caso. Deve existir no mínimo uma unidade de notificação por município (9).

O tempo de investigação do caso, por sua vez, é considerado adequado quando $80 \%$ ou mais dos casos de PFA notificados são investigados dentro das 48 horas posteriores à notificação. Considerando a apresentação clínica aguda e as características de transmissão do poliovírus selvagem $(9,12)$, esse tempo de análise do caso é essencial para garantir a eficiência do diagnóstico clínico (avaliação precoce dos sintomas e manifestações físicas) e possibilitar a coleta de exames diagnósticos.

O indicador de investigação diagnóstica é considerado adequado se $80 \%$ ou mais dos casos de PFA notificados têm coletadas duas amostras de fezes para cultura viral nas 2 semanas seguintes ao início da deficiência motora (9).

As definições de "caso" (suspeito, provável, confirmado, compatível, associado à vacina e descartado) foram descritas nas diretrizes da OPAS (13).

Os dados referentes às áreas territoriais das 27 unidades federadas e regiões geográficas ${ }^{2}$ do Brasil foram extraídos da base de dados da Fundação Instituto Brasileiro de Geografia e Estatística (IBGE) (14). Tais dados foram utilizados para inferir possíveis diferenças entre áreas e indicadores de efetividade.

No banco de dados do estudo, o valor de cada indicador foi registrado por ano e por unidade federada, de acordo com o modelo utilizado pela FUNASA. Os valores foram totalizados também por região geográfica do

\footnotetext{
2 Denominação das regiões e unidades federadas do território brasileiro. Norte: Acre (AC), Amapá (AP), Amazonas (AM), Pará (PA), Rondônia (RO), Roraima (RR) e Tocantins (TO); Nordeste: Alagoas (AL), Bahia (BA), Ceará (CE), Maranhão (MA), Paraíba (PB), Pernambuco (PE),Piauí (PI), Rio Grande do Norte (RN) e Sergipe (SE); Sudeste: Espírito Santo (ES), Minas Gerais (MG), Rio de Janeiro (RJ) e São Paulo (SP); Centro-Oeste: Distrito Federal (DF), Goiás (GO), Mato Grosso (MT) e Mato Grosso do Sul (MS); Sul: Paraná (PR), Rio Grande do Sul (RS) e Santa Catarina (SC).
} 
TABELA 1. Prevalência de casos de paralisias flácidas agudas entre 100000 menores de 15 anos, Brasil, 1990 a $2000^{a}$

\begin{tabular}{|c|c|c|c|c|c|c|c|c|c|c|c|c|c|}
\hline \multirow[b]{2}{*}{ Média } & \multicolumn{5}{|c|}{ Pré-certificação } & \multirow[b]{2}{*}{ Unidade federada } & \multirow[b]{2}{*}{1995} & \multicolumn{6}{|c|}{ Pós-certificação } \\
\hline & 1990 & 1991 & 1992 & 1993 & 1994 & & & 1996 & 1997 & 1998 & 1999 & 2000 & Média \\
\hline $1,4^{b}$ & $2,5^{b}$ & 0 & $1,6^{b}$ & $1^{b}$ & 2 & Acre & 0,5 & $1,9^{b}$ & $1^{b}$ & 0,5 & 0 & $1^{b}$ & 0,8 \\
\hline $1,4^{b}$ & $2,8^{b}$ & $1,3^{b}$ & $1,7^{b}$ & 0,6 & 0,5 & Alagoas & 0,5 & $1,2^{b}$ & 0,9 & 0,6 & $1^{b}$ & $1^{\mathrm{b}}$ & 0,9 \\
\hline $1,5^{\mathrm{b}}$ & $1^{b}$ & $1,6^{b}$ & $2,2^{b}$ & 0,7 & $2,1^{b}$ & Amapá & 0,7 & $1,9^{b}$ & $1,3^{b}$ & $1^{b}$ & 0,5 & $3,2^{b}$ & $1,4^{b}$ \\
\hline $1,1^{b}$ & $1,6^{b}$ & 0,9 & $1,2^{b}$ & 0,5 & $1,5^{b}$ & Amazonas & $1,8^{b}$ & $1,5^{b}$ & $1,5^{b}$ & $1,5^{b}$ & $2,8^{b}$ & $1,4^{b}$ & $1,8^{b}$ \\
\hline $1,3^{b}$ & $1,9^{b}$ & $1,3^{b}$ & $1,1^{b}$ & $1,3^{b}$ & $1,1^{\mathrm{b}}$ & Bahia & 0,8 & 0,8 & 0,7 & 0,6 & 0,8 & $1^{b}$ & 0,8 \\
\hline $1^{b}$ & $1,4^{b}$ & $1,1^{b}$ & $1^{b}$ & 0,8 & 0,9 & Ceará & 0,7 & 0,8 & 0,9 & 0,4 & $1^{\mathrm{b}}$ & $1^{b}$ & 0,8 \\
\hline $2,3^{b}$ & $3,1^{\mathrm{b}}$ & $3^{b}$ & $1,7^{\mathrm{b}}$ & $1,5^{\mathrm{b}}$ & $2,3^{b}$ & Distrito Federal & $1^{b}$ & $3,1^{b}$ & $1,7^{b}$ & $2,4^{b}$ & $1,1^{b}$ & $1^{b}$ & $1,7^{b}$ \\
\hline $1,1^{\mathrm{b}}$ & 0,9 & $1,1^{b}$ & $1,5^{b}$ & $1,3^{b}$ & 0,8 & Espírito Santo & $1,3^{b}$ & $1,3^{b}$ & 0,9 & 0,4 & $1,3^{b}$ & $1^{b}$ & $1^{b}$ \\
\hline $1,2^{b}$ & $1,3^{b}$ & 0,8 & $1^{b}$ & $1,1^{b}$ & $1,7^{\mathrm{b}}$ & Goiás & 0,9 & 0,7 & 0,9 & 0,8 & 0,8 & $1^{b}$ & 0,9 \\
\hline 0,7 & 0,6 & 0,8 & $1^{b}$ & 0,7 & 0,5 & Maranhão & 0,4 & 0,3 & 0,4 & 0,3 & 0,5 & 0,9 & 0,5 \\
\hline $1,1^{b}$ & 0,9 & $2,1^{b}$ & 0,9 & 0,8 & 0,6 & Mato Grosso & $1,2^{b}$ & 0,7 & 0,8 & 0,5 & 0,7 & $1^{b}$ & 0,8 \\
\hline $1,1^{b}$ & $1,2^{b}$ & 0,5 & $1,8^{b}$ & 0,6 & $1,3^{b}$ & Mato Grosso do Sul & $1^{b}$ & 0,4 & 0,3 & 0,9 & 0,9 & $1^{b}$ & 0,8 \\
\hline 0,8 & 0,5 & 0,9 & 0,8 & 0,8 & $1,2^{b}$ & Minas Gerais & 0,8 & 0,5 & 0,6 & 0,6 & 0,7 & $1^{b}$ & 0,7 \\
\hline $1,2^{b}$ & $1,7^{b}$ & 0,9 & 0,9 & 0,8 & $1,5^{b}$ & Pará & $1,3^{b}$ & $1,2^{b}$ & 0,8 & 0,7 & 0,8 & 0,7 & 0,9 \\
\hline $1,1^{b}$ & $1,8^{b}$ & $1^{b}$ & 0,9 & $1,1^{b}$ & 0,7 & Paraíba & 0,5 & 0,8 & $1^{b}$ & 0,6 & 0,7 & $1^{\mathrm{b}}$ & 0,8 \\
\hline $1,2^{b}$ & $1,3^{b}$ & $1,2^{b}$ & $1,4^{b}$ & $1,1^{\mathrm{b}}$ & $1,2^{b}$ & Paraná & $1,1^{\mathrm{b}}$ & $1,2^{b}$ & $1^{b}$ & 0,7 & 0,4 & $1^{b}$ & 0,9 \\
\hline 0,9 & 0,8 & $1,2^{b}$ & $1,1^{\mathrm{b}}$ & $1,1^{b}$ & 0,5 & Pernambuco & 0,4 & 0,8 & 0,8 & 0,9 & $1,3^{b}$ & $1,2^{b}$ & 0,9 \\
\hline 0,8 & 0,4 & 0,8 & $1^{b}$ & 0,9 & $1,1^{b}$ & Piauí & $1^{b}$ & 0,8 & $1^{b}$ & 0,6 & 0,5 & $1^{b}$ & 0,8 \\
\hline 0,7 & 0,3 & 0,7 & 0,9 & 0,8 & 0,7 & Rio de Janeiro & 0,8 & 0,9 & $1,2^{b}$ & 0,7 & $1^{\mathrm{b}}$ & $1,1^{b}$ & $1^{\mathrm{b}}$ \\
\hline $1,6^{b}$ & $1,7^{\mathrm{b}}$ & $1,8^{b}$ & $2^{b}$ & $1,1^{b}$ & $1,2^{b}$ & Rio Grande do Norte & $1,2^{b}$ & 0,8 & $1^{b}$ & $1,1^{\mathrm{b}}$ & $1^{b}$ & 0,8 & $1^{b}$ \\
\hline $2,4^{b}$ & 0,3 & 0,9 & 0,9 & 9 & $1,1^{\mathrm{b}}$ & Rio Grande do Sul & $1^{b}$ & 0,8 & 0,9 & $1,3^{b}$ & 0,8 & $1,7^{b}$ & $1,1^{b}$ \\
\hline $1,7^{b}$ & $1,3^{b}$ & $1,7^{b}$ & 0,9 & $1,8^{b}$ & $2,7^{\mathrm{b}}$ & Rondônia & $1,3^{b}$ & 1 & 0,9 & 0,4 & $2^{b}$ & $1^{b}$ & $1,1^{\mathrm{b}}$ \\
\hline $2,1^{b}$ & $6,5^{\mathrm{b}}$ & 0 & 0 & 0 & $4,1^{\mathrm{b}}$ & Roraima & 0 & $1^{b}$ & 0 & 0 & $2^{b}$ & 0 & 0,5 \\
\hline 0,9 & 0,8 & 0,9 & $1^{b}$ & $1^{b}$ & 0,8 & Santa Catarina & 0,9 & 0,6 & 0,9 & 0,5 & $1^{b}$ & 0,9 & 0,8 \\
\hline 0,7 & 0,5 & 0,9 & 0,7 & 0,7 & 0,8 & São Paulo & 0,4 & 0,6 & 0,4 & 0,5 & 0,5 & 0,7 & 0,5 \\
\hline $1,8^{b}$ & $4,5^{b}$ & $1,3^{b}$ & $1,3^{b}$ & 0,9 & $1,1^{\mathrm{b}}$ & Sergipe & 0,6 & $1,1^{\mathrm{b}}$ & $1,1^{\mathrm{b}}$ & 0,1 & $1,6^{b}$ & $3,9^{b}$ & $1,4^{b}$ \\
\hline 0,7 & 0,2 & 0,7 & 1,1 & 0,8 & 0,7 & Tocantins & 0 & $1,4^{b}$ & $1,1^{\mathrm{b}}$ & 0,5 & $1,4^{b}$ & 0,8 & 0,9 \\
\hline \multicolumn{14}{|c|}{ País e regiões } \\
\hline $1,3^{b}$ & $1,5^{b}$ & $1,1^{b}$ & $1,2^{b}$ & $1,2^{b}$ & $1,3^{b}$ & Brasil & 0,8 & $1^{b}$ & 0,9 & 0,7 & $1^{b}$ & $1,2^{b}$ & 0,9 \\
\hline $1,5^{\mathrm{b}}$ & $2,4^{b}$ & 0,9 & $1,1^{b}$ & 0,8 & $2,3^{b}$ & Norte & 0,9 & $1,4^{b}$ & 0,9 & 0,7 & $1,4^{b}$ & $1,2^{b}$ & $1,1^{\mathrm{b}}$ \\
\hline $1,3^{b}$ & $1,3^{b}$ & $1,4^{b}$ & $1,3^{b}$ & $1,0^{b}$ & $1,3^{b}$ & Centro Oeste & 0,8 & $1,3^{b}$ & $1,0^{b}$ & $1,0^{b}$ & $1,0^{b}$ & $1,0^{b}$ & $1,0^{b}$ \\
\hline $1,2^{b}$ & $1,8^{b}$ & $1,2^{b}$ & $1,2^{b}$ & 0,9 & 0,8 & Nordeste & 0,7 & 0,8 & 0,9 & 0,6 & 0,9 & $1,3^{b}$ & 0,9 \\
\hline 0,8 & 0,6 & 0,9 & $1,0^{b}$ & 0,9 & 0,9 & Sudeste & 0,8 & 0,8 & 0,8 & 0,6 & 0,9 & $1,0^{b}$ & 0,8 \\
\hline $1,5^{\mathrm{b}}$ & 0,8 & $1,0^{b}$ & $1,1^{\mathrm{b}}$ & $3,7^{b}$ & $1,0^{b}$ & Sul & $1,0^{b}$ & 0,9 & 0,9 & 0,8 & 0,7 & $1,2^{b}$ & 0,9 \\
\hline
\end{tabular}

a Prevalência antes e depois da certificação do Brasil como zona livre de poliomielite.

b Valores considerados adequados (notificação $\geq 1$ caso em 100000 indivíduos com menos de 15 anos de idade).

Brasil. A partir dos pontos de corte de cada indicador, acima referidos, cada valor observado foi classificado em duas categorias: 1) adequado, se igual ou superior ao valor considerado como efetivo; ou 2) inadequado, se abaixo do valor mínimo estabelecido.

A razão percentual foi uma variável construída pelo valor agregado dos indicadores em relação ao tempo. Essa variável foi desenvolvida para ser mais expressiva do que as medidas de tendência central convencionais (média e mediana). Utilizando a relação efetividade/tempo para cada unidade, aplicou-se a técnica da razão percentual, pois esta torna-se matematicamente mais apta para expressar a efetividade total por período, considerando o valor binário (efetivo/não efetivo). Considerando-se que $100 \%$ de efetividade ocorreria se fosse atingida a meta na totalidade das unidades federadas, em todos os anos do estudo, foi calculada a razão percentual de valores adequados verdadeiramente alcançados em cada unidade federada em cada ano, para cada período do estudo. Também foram representadas as médias finais por período, unidade e região, mantendo-se a mesma metodologia de apresentação utilizada na documentação oficial da OMS e OPAS para acompanhamento do programa (1). Utilizamos as médias finais para comparação entre os dois períodos de estudo.

$\mathrm{O}$ teste do qui-quadrado $\left(\chi^{2}\right)$ e o teste exato de Fisher foram utiliza- dos para avaliar a diferença entre as variáveis nos períodos pré e póscertificação. A diferença foi considerada significativa para um valor de probabilidade $(\alpha)$ menor ou igual a $5 \%$ $(P \leq 0,05)$.

\section{RESULTADOS}

A tabela 1 mostra os resultados para o indicador prevalência mínima. As médias finais de prevalência para cada período de estudo mostram valores adequados em 19 (70,4\%) das 27 unidades federadas no período pré-certificação, e em apenas nove $(33,3 \%$, ) no período pós-certificação, sendo essa diferença estatisticamente significativa 
FIGURA 1. Razões percentuais de efetividade dos indicadores de qualidade de vigilância epidemiológica para paralisias flácidas agudas antes e depois da eliminação da poliomielite, Brasil, 1990 a 2000

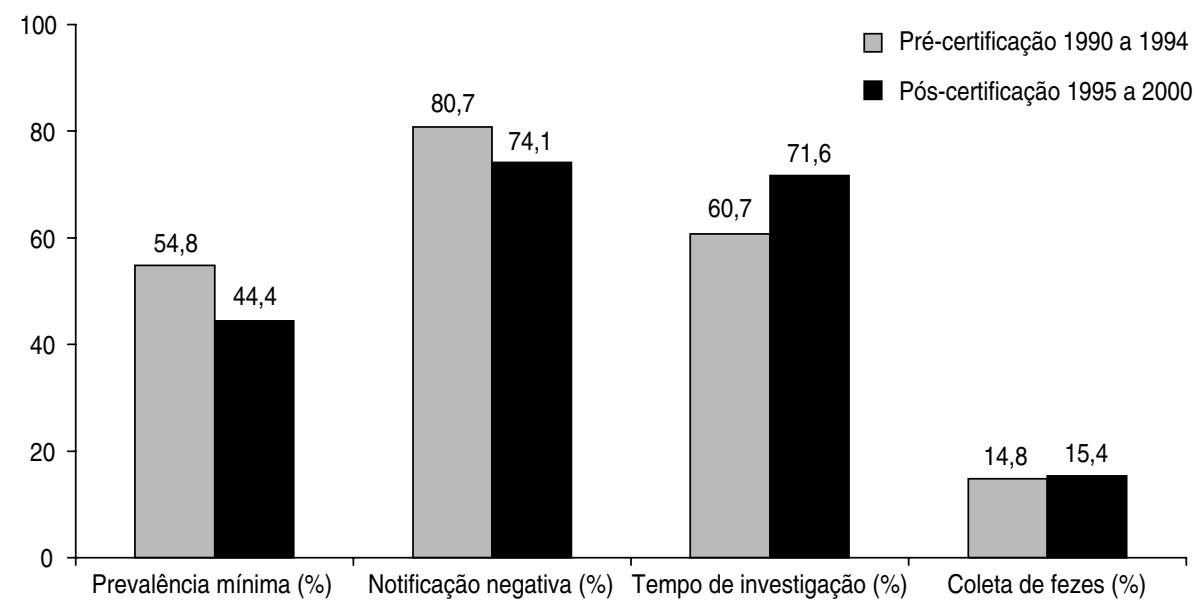

$(P<0,014)$. Em termos de razão percentual (figura 1), houve uma redução de $10,4 \%$ em termos de efetividade para esse indicador, porém sem significância estatística $(P>0,08)$.

Ainda sobre prevalência mínima, no período pré-certificação apenas o Distrito Federal e os Estados da Bahia, Paraná e Rio Grande do Norte foram $100 \%$ efetivos. Após a certificação, apenas o Distrito Federal e o Estado do Amazonas mantiveram a mesma efetividade. Esses têm, respectivamente, a menor e a maior área territorial, e a maior e a segunda menor densidade populacional no país (14). A média da Região Norte (maior extensão territorial), nos dois períodos, foi efetiva,

TABELA 2. Notificação negativa semanal de casos de paralisias flácidas agudas, Brasil, 1990 a $2000^{a}$

\begin{tabular}{|c|c|c|c|c|c|c|c|c|c|c|c|c|c|c|}
\hline \multirow[b]{2}{*}{ Média } & \multicolumn{5}{|c|}{ Pré-certificação } & \multirow[b]{2}{*}{ Unidade federada } & \multirow[b]{2}{*}{$\%$ áreab $^{b}$} & \multicolumn{7}{|c|}{ Pós-certificação } \\
\hline & 1990 & 1991 & 1992 & 1993 & 1994 & & & 1995 & 1996 & 1997 & 1998 & 1999 & 2000 & Média \\
\hline $86^{c}$ & 60 & $91^{\mathrm{c}}$ & $95^{\mathrm{C}}$ & $91^{c}$ & $95^{\mathrm{c}}$ & Acre & 1,79 & $95^{c}$ & $91^{\mathrm{c}}$ & 65 & $91^{\mathrm{C}}$ & 71 & 63 & 79 \\
\hline $88^{c}$ & 78 & $89^{c}$ & $92^{c}$ & $93^{c}$ & $89^{c}$ & Amapá & 1,68 & $88^{c}$ & $100^{c}$ & $100^{c}$ & $100^{c}$ & $94^{\mathrm{c}}$ & $91^{\mathrm{c}}$ & $96^{c}$ \\
\hline $97^{c}$ & $96^{c}$ & $97^{\mathrm{c}}$ & $98^{\mathrm{c}}$ & $94^{\mathrm{C}}$ & $100^{c}$ & Amazonas & 18,46 & $85^{c}$ & $91^{\mathrm{c}}$ & $93^{c}$ & 73 & 66 & $92^{\mathrm{c}}$ & $83^{c}$ \\
\hline $98^{c}$ & $95^{c}$ & $97^{c}$ & $99^{c}$ & $100^{c}$ & $98^{\mathrm{C}}$ & Bahia & 6,63 & $100^{c}$ & $98^{c}$ & $100^{c}$ & 73 & $81^{c}$ & $85^{c}$ & $90^{c}$ \\
\hline $87^{c}$ & 63 & $92^{\mathrm{c}}$ & $98^{\mathrm{c}}$ & $95^{c}$ & $87^{c}$ & Ceará & 1,71 & $86^{c}$ & $95^{c}$ & $92^{c}$ & $92^{c}$ & $95^{\mathrm{c}}$ & $92^{c}$ & $92^{\mathrm{c}}$ \\
\hline $94^{c}$ & $95^{c}$ & $91^{\mathrm{c}}$ & $99^{c}$ & $98^{\mathrm{C}}$ & $89^{c}$ & Goiás & 4,00 & $95^{\mathrm{C}}$ & $98^{\mathrm{C}}$ & $83^{c}$ & $98^{\mathrm{C}}$ & $85^{c}$ & 78 & $90^{c}$ \\
\hline $84^{c}$ & 77 & $90^{c}$ & $89^{c}$ & $83^{c}$ & $83^{c}$ & Maranhão & 3,90 & 72 & 79 & 77 & 51 & 61 & $86^{c}$ & 71 \\
\hline $83^{c}$ & 71 & $85^{c}$ & $91^{\mathrm{c}}$ & 73 & $93^{c}$ & Mato Grosso & 10,61 & $100^{c}$ & $98^{\mathrm{c}}$ & $100^{c}$ & 76 & 68 & $94^{\mathrm{C}}$ & $89^{c}$ \\
\hline $96^{c}$ & $96^{c}$ & $91^{\mathrm{c}}$ & $100^{c}$ & $96^{c}$ & $97^{c}$ & Mato Grosso do Sul & 4,20 & 78 & $83^{c}$ & $88^{c}$ & $97^{c}$ & $100^{c}$ & $94^{\mathrm{C}}$ & $90^{c}$ \\
\hline 78 & $80^{c}$ & 56 & 78 & $83^{c}$ & $91^{\mathrm{c}}$ & Minas Gerais & 6,89 & $91^{c}$ & $84^{c}$ & 79 & $81^{\mathrm{C}}$ & 71 & 71 & $80^{\circ}$ \\
\hline $87^{\circ}$ & $89^{c}$ & $93^{c}$ & $91^{\mathrm{c}}$ & 76 & $87^{c}$ & Pará & 14,66 & $90^{c}$ & $94^{c}$ & $94^{\mathrm{c}}$ & $94^{\mathrm{C}}$ & $89^{c}$ & $82^{c}$ & $91^{c}$ \\
\hline $89^{c}$ & $98^{\mathrm{c}}$ & $80^{c}$ & $92^{c}$ & $84^{c}$ & $93^{c}$ & Rio de Janeiro & 0,51 & $100^{c}$ & $98^{\mathrm{c}}$ & $100^{c}$ & $96^{c}$ & $88^{c}$ & $94^{c}$ & $96^{c}$ \\
\hline $96^{c}$ & $91^{c}$ & $95^{\mathrm{c}}$ & $98^{\mathrm{c}}$ & $98^{\mathrm{c}}$ & $97^{\mathrm{c}}$ & Rio Grande do Norte & 0,62 & $96^{c}$ & $98^{\mathrm{c}}$ & $98^{c}$ & $97^{c}$ & $97^{c}$ & 77 & $94^{c}$ \\
\hline $96^{c}$ & $93^{c}$ & $92^{c}$ & $97^{c}$ & $96^{c}$ & $100^{c}$ & Rio Grande do Sul & 3,31 & $100^{c}$ & $97^{c}$ & $100^{c}$ & $97^{c}$ & 43 & $97^{c}$ & $89^{c}$ \\
\hline $92^{c}$ & $93^{c}$ & $84^{c}$ & $97^{c}$ & $89^{c}$ & $98^{\mathrm{C}}$ & Rondônia & 2,79 & $98^{c}$ & 67 & $97^{c}$ & $91^{\mathrm{c}}$ & $81^{c}$ & 65 & $83^{c}$ \\
\hline $88^{c}$ & $80^{c}$ & $100^{c}$ & $80^{c}$ & $84^{c}$ & $94^{c}$ & Roraima & 2,63 & $90^{c}$ & $89^{c}$ & 67 & 55 & $69^{c}$ & $81^{\mathrm{C}}$ & 75 \\
\hline $93^{c}$ & $99^{c}$ & $80^{c}$ & $97^{c}$ & $96^{c}$ & $95^{c}$ & Santa Catarina & 1,12 & $96^{c}$ & $93^{c}$ & $96^{c}$ & 77 & $95^{\mathrm{c}}$ & $87^{\circ}$ & $91^{c}$ \\
\hline $83^{c}$ & 41 & $92^{\mathrm{c}}$ & $92^{\mathrm{c}}$ & $93^{c}$ & $96^{c}$ & São Paulo & 2,92 & $98^{\mathrm{c}}$ & $99^{c}$ & $100^{c}$ & $96^{c}$ & $92^{\mathrm{c}}$ & $88^{c}$ & $96^{c}$ \\
\hline 72 & 64 & $86^{c}$ & $91^{\mathrm{c}}$ & $92^{\mathrm{C}}$ & 27 & Sergipe & 0,26 & $100^{c}$ & $100^{c}$ & $100^{c}$ & 70 & $83^{c}$ & $99^{c}$ & $92^{c}$ \\
\hline \multirow[t]{2}{*}{76} & 63 & 62 & 73 & $92^{c}$ & $91^{\mathrm{c}}$ & Tocantins & 3,26 & 57 & 66 & 72 & 77 & $95^{c}$ & $93^{c}$ & 77 \\
\hline & & & & & & País e regiões & & & & & & & & \\
\hline $87^{\circ}$ & $82^{c}$ & $88^{c}$ & $92^{\mathrm{c}}$ & $90^{c}$ & $85^{c}$ & Brasil & 100,0 & $90^{c}$ & $88^{c}$ & $87^{c}$ & 79 & 77 & $85^{c}$ & $84^{c}$ \\
\hline $90^{c}$ & $83^{c}$ & $92^{\mathrm{c}}$ & $92^{\mathrm{c}}$ & $88^{c}$ & $94^{c}$ & Norte & 42,01 & $91^{\mathrm{c}}$ & $89^{c}$ & $86^{c}$ & $84^{c}$ & 78 & 79 & $84^{c}$ \\
\hline $90^{c}$ & $85^{c}$ & $86^{c}$ & $93^{c}$ & $92^{c}$ & $94^{c}$ & Centro Oeste & 22,14 & $86^{c}$ & $89^{c}$ & $89^{c}$ & $89^{c}$ & $90^{c}$ & $92^{\mathrm{c}}$ & $89^{\circ}$ \\
\hline
\end{tabular}

a Porcentagem antes e depois da certificação do Brasil como zona livre de poliomielite.

${ }^{b}$ Em relação ao território do Brasil (8 $\left.511227,2 \mathrm{~km}^{2}\right)$.

${ }^{c}$ Valores considerados adequados (ausência de casos informada semanalmente por no mínimo $80 \%$ das unidades notificadoras em cada região). 
TABELA 3. Tempo de investigação de casos de paralisias flácidas agudas, Brasil, 1990 a $2002^{a}$

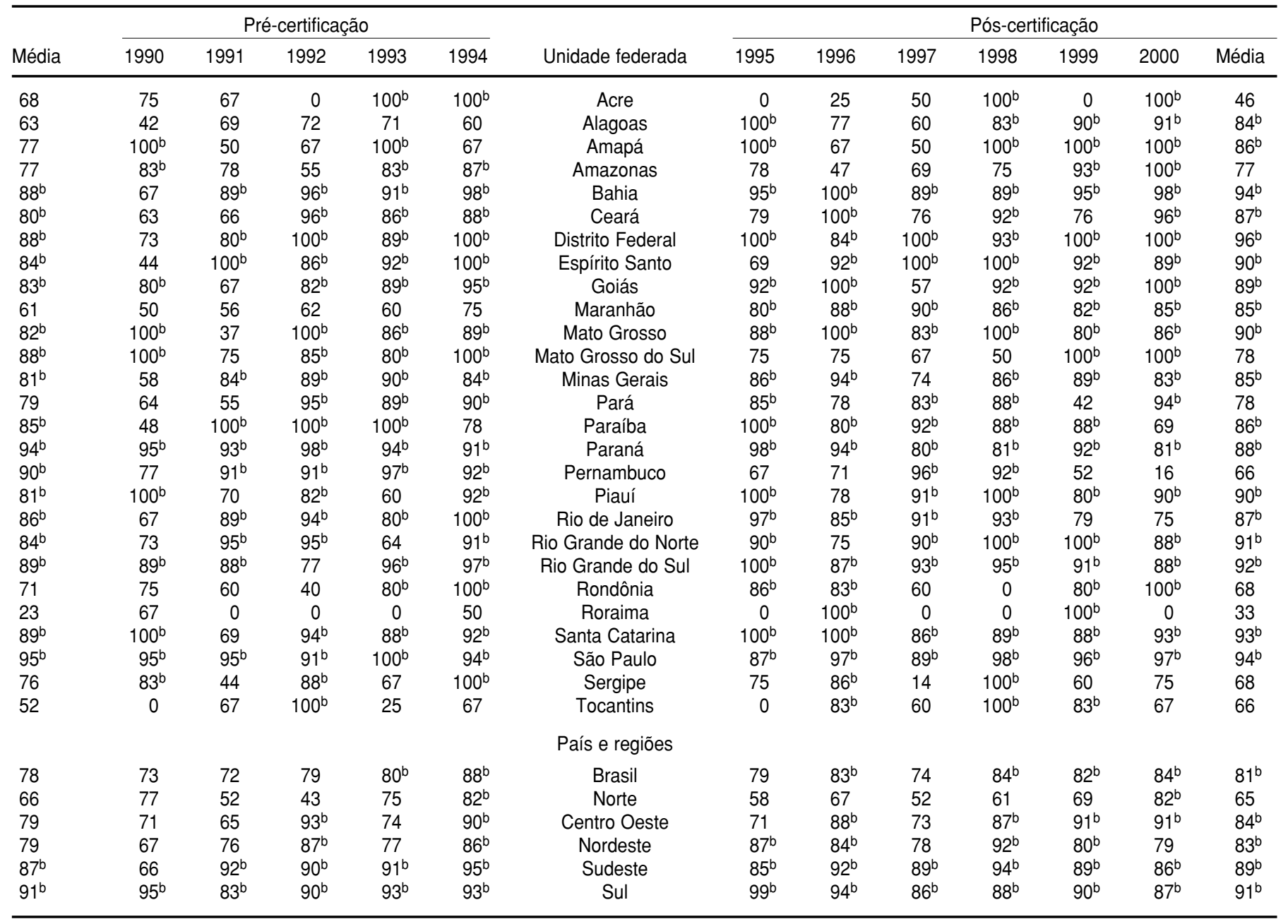

a Porcentagem antes e depois da certificação do Brasil como zona livre de poliomielite.

b Valores considerados adequados (investigação de $80 \%$ ou mais dos casos notificados dentro das 48 horas posteriores à notificação).

com valores pré e pós-certificação de 1,5 e 1,1 por 100000 menores de 15 anos. A medida da média simples demonstrou efetividade, embora os valores fossem inadequados em $5(45,5 \%)$ dos 11 anos estudados.

Quanto à notificação negativa semanal, a tabela 2 evidencia valores adequados em $22(81,5 \%)$ das 27 unidades federadas no período précertificação. Após a certificação, 20 $(71,4 \%)$ unidades federadas apresentaram valores adequados $(P>0,74)$. Esses valores, entretanto, devem ser examinados considerando-se as diferenças no tamanho do território das unidades federadas. Assim, observase que o Distrito Federal, que tem o menor território, obteve o melhor de- sempenho em todo o período do estudo, seguido pelo Paraná e pelo Rio de Janeiro. Comparando as unidades com área territorial $\leq 3 \%$ do território nacional $(\mathrm{n}=17)$ com aquelas cujo território é > 3\% do território nacional $(\mathrm{n}=10)$, no período pré certificação as freqüências de valores adequados foram respectivamente de $82,4 \%$ $(14 / 17)$ e $80,0 \%(8 / 10)(P>0,62)$; a mesma análise, no período pós-certificação, apresentou freqüências, respectivamente, de $70,6 \%(12 / 17)$ e $80 \%$ $(8 / 10)(P>0,47)$. No que diz respeito à razão percentual de efetividade por período para esse indicador (figura 1), a redução de $6,6 \%$ entre os dois períodos não alcançou significância estatística $(P>0,21)$.
A tabela 3 mostra a evolução da efetividade do tempo de investigação dos casos notificados nos dois períodos de estudo. Das 27 unidades federadas, 17 $(63 \%)$ alcançaram níveis médios finais adequados antes da certificação e 66,7\% $(\mathrm{n}=18)$ depois da certificação $(P>0,99)$. Quanto à razão percentual de efetividade por período para este indicador (figura 1), houve um aumento de 10,9\%. Essa diferença ficou próxima ao limite de significância estatística $(P>0,06)$.

$\mathrm{O}$ indicador referente à investigação diagnóstica (tabela 4) manteve-se dentro de valores inadequados tanto no período pré $(54 \%)$ quanto póscertificação (52\%). De 1990 a 1994, somente o Distrito Federal alcançou média de efetividade $(84 \%)$. A razão 
TABELA 4. Coleta de duas amostras adequadas de fezes nas primeiras 2 semanas do início das paralisias flácidas agudas, Brasil, 1990 a $2000^{2}$

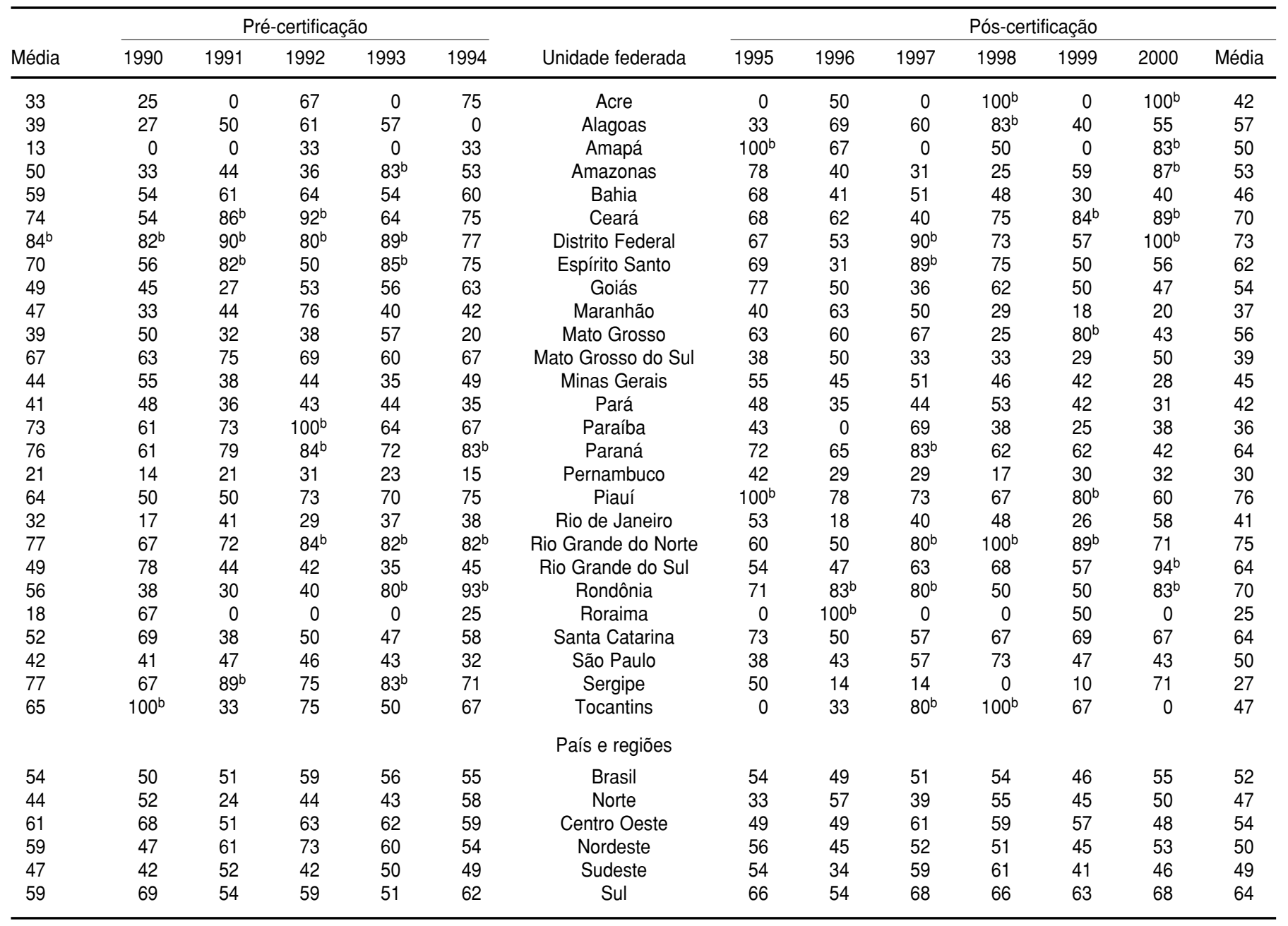

a Porcentagem antes e depois da certificação do Brasil como zona livre de poliomielite.

${ }^{b}$ Valores considerados adequados (coleta de duas amostras de fezes para cultura viral nas 2 semanas seguintes ao início da deficiência motora em $80 \%$ ou mais dos casos).

percentual de efetividade para este indicador (figura 1) foi de $14,8 \%$ antes da certificação e $15,4 \%$ após a certificação $(P>0,99)$.

\section{DISCUSSÃO}

O processo de vigilância epidemiológica adotado no Brasil foi estabelecido por lei em 1990, e está descrito (9) como "um conjunto de ações que proporciona o conhecimento, a detecção ou prevenção de qualquer mudança nos fatores determinantes e condicionantes de saúde individual ou coletiva, com a finalidade de recomendar e adotar as medidas de prevenção e controle das doenças ou agravos" (p. 12). Segundo o
Manual de Vigilância Epidemiológica da FUNASA (9), indicador é uma "informação produzida com periodicidade definida e critérios constantes" (p. $60)$. Os indicadores são variáveis suscetíveis à mensuração direta, que refletem indiretamente o estado de saúde de pessoas em uma comunidade (15). A adoção de indicadores para monitorar o programa de erradicação mundial da poliomielite por vírus selvagem foi uma opção necessária para que existissem mecanismos padronizados de controle dos processos. Isso permitiu a geração de informação com qualidade nas diversas regiões do mundo, com representações passíveis de interpretação, de modo uniforme, pelos coordenadores do programa.
Dentre os indicadores de vigilância epidemiológica para paralisias flácidas agudas, a prevalência mínima de casos foi considerada no Brasil como o indicador mais relevante (16). Ao se observar, no presente estudo, a redução que ocorreu na média final no período pós-certificação (0,9 por 100000 casos em menores de 15 anos), associada à queda de desempenho da razão percentual de efetividade das unidades federadas $(-10,4 \%)$, percebese a necessidade de melhorar a metodologia de detecção dos casos, seja por campanhas de esclarecimento, seja por novos instrumentos de vigilância ativa e busca direta.

Entretanto, o parâmetro de controle da doença que é mais conhecido e di- 
vulgado para a população ainda é a cobertura vacinal. Por isso, muitos profissionais de saúde ignoram a importância da vigilância epidemiológica e da notificação de PFA no processo de controle da poliomielite. Nesse sentido, as campanhas educativas têm sido uma alternativa eficaz para impulsionar a melhoria da qualidade dos indicadores. $\mathrm{Na}$ Índia e no oeste da África, por exemplo, uma campanha de conscientização dos curandeiros e líderes religiosos elevou significativamente a taxa de prevalência mínima (17). A vigilância passiva, portanto, necessita adaptar-se às realidades sócio-culturais do meio em que atua, pois a colaboração da população e da comunidade médica é essencial para a efetividade do sistema de notificação de doenças (15). Para a realidade brasileira, uma nova oportunidade de aprimorar o indicador da prevalência mínima está ocorrendo com a ampliação do programa de agentes comunitários de saúde, os quais, se corretamente orientados, poderão ampliar a capacidade de detectar casos de PFA.

A monitoração dos eventos "poliomielite" e "paralisias flácidas agudas" pelo sistema de informações hospitalares do Sistema Único de Saúde (SUS) (18) também tem auxiliado na busca ativa de casos de pólio. A utilização dos diagnósticos de internação facilitou a prospecção de casos, pois o cruzamento das informações é mais uma ferramenta de vigilância à prevalência mínima. A partir de janeiro de 1998, com a adoção da décima revisão da classificação internacional de doenças e causas de morte (CID-10), que melhor discrimina essas denominações, foi possível a comparação mais acurada entre os números de casos notificados em cada região e aqueles internados em unidades de saúde, melhorando assim o fluxo de dados (18).

O sistema de informações sobre agravos de notificação (SINAN) é um banco de dados centralizado e unificado para todo o território brasileiro. O SINAN vem sendo desenvolvido desde 1992, mas ainda não está plenamente em operação (19). Após a implantação plena desse sistema, o fluxo e a disponibilidade das informações certamente promoverão a detecção e a monitoração dos casos de PFA, desde que seja estabelecido um programa contínuo de busca ativa em todos os bancos de dados do SINAN, visando a fornecer informações a curto prazo para a vigilância das PFA. Desse modo, haverá a possibilidade de monitoração contínua, e não apenas a implantação de algum mecanismo suplementar quando o indicador se apresentar inadequado.

A notificação negativa semanal foi instituída no Brasil em 1990, no mesmo ano em que se estabeleceram os critérios de concessão da certificação para as Américas (2). Esse indicador tem por objetivo o registro fiel da não ocorrência de casos, e deve ser interpretado conjuntamente com o valor da prevalência mínima. Havendo a baixa efetividade dos dois indicadores, devese considerar o sistema em alerta para o risco de reintrodução do poliovírus. Os avanços nos sistemas de comunicação e integração de dados dos sistemas de saúde, acima referidos, deverão elevar a efetividade de notificação negativa nos próximos anos.

$\mathrm{O}$ indicador da agilidade do processo de investigação dos casos evidencia também a capacidade dos recursos humanos e dos serviços de uma área ou região territorial de avaliar o caso e adotar as medidas de controle. Sendo assim, é o primeiro contato com a estrutura capacitada para a classificação do caso, daí ser essencial ao gerenciamento do sistema de vigilância, devendo ocorrer nas primeiras 48 horas após a comunicação. Nesse momento, com base no quadro clínico (12), o sistema já tem capacidade de decisão para prosseguir com a investigação e deflagrar, quando necessário, uma operação de bloqueio de potencial epidemia. A classificação precoce do tipo viral também permite uma maior especificidade nas ações de contenção.

Apesar de haver eliminação do vírus e potencial detecção do mesmo até 4 semanas após o início dos sintomas, a positividade da cultura de fezes é maior nas 2 primeiras semanas (20). Por isso, o indicador de diagnóstico mensurado pela coleta adequada de fezes nas primeiras 2 semanas do início da paralisia reflete a capacidade de diagnóstico de uma área ou região territorial, sendo também um indicador de biossegurança para a não ocorrência do poliovírus selvagem (21). Em paralelo, esse indicador auxilia na investigação diagnóstica de outras condições que possam manifestar PFA. Apesar disso, esse indicador manteve valores médios inadequados em ambos os períodos estudados (54 e 52\%) Entretanto, no processo de certificação do Brasil, a ausência de casos de infecção pelo vírus selvagem foi o mais relevante indicador utilizado na avaliação do sistema de vigilância.

$\mathrm{Na}$ rede de laboratórios oficiais do sistema de vigilância das Américas, ficou evidenciado que uma única amostra de fezes, coletada nas 2 primeiras semanas de paralisia, pode ser suficiente para a detecção satisfatória da circulação do poliovírus (22). Também foi demonstrado que a investigação das amostras fecais dos contatos, como se recomendava no início da implantação do sistema de vigilância, tinha pequena sensibilidade para detectar o poliovírus na comunidade (20). Esses dados sugerem que, em áreas endêmicas, seria possível considerar a coleta de apenas uma amostra de fezes para diagnóstico, e que, apenas em circunstâncias excepcionais, como, por exemplo, a pesquisa de vírus vacinal, deveriam ser coletadas amostras de contatos. Essa proposta simplifica a investigação dos casos, reduz a carga de trabalho dos laboratórios e coletores e aumenta a eficiência geral do sistema (23).

Em publicação normativa da OPAS (10), de junho de 1999, reduziu-se para uma amostra a determinação para esse indicador de diagnóstico; entretanto, o sistema brasileiro manteve a recomendação das duas amostras. No relatório da comissão de certificação para as Américas, em 2001, registrou-se que o Brasil alcançou, de 1995 a 2000, uma média de $54,5 \%$ para esse indicador (24). Em comparação com os países com os quais faz fronteira, o Brasil superou apenas a Argentina, que alcançou $40 \%$.

Estruturar e manter uma vigilância epidemiológica adequada é, após a in- 
terrupção na transmissão do vírus, o principal fator para alcançar-se a erradicação da pólio em nível mundial (25). Ao contrário dos 2 dias nacionais de imunização no Brasil, que são festivos, populares e de curta duração, a vigilância epidemiológica é de instalação lenta e esforço contínuo, exigindo perseverança e investimento das instituições estatais. Quanto menos estruturado é o sistema de saúde pública de um país, mais complicado e ineficaz é o processo de edificação de uma vigilância epidemiológica satisfatória (26). Por isso, a OMS (27) alerta para a necessidade de aprimoramento dessas ações, e chama a atenção para os riscos de reintrodução do vírus selvagem da poliomielite em áreas consideradas livres.

\section{CONCLUSÕES}

As novas estratégias de informação que estão sendo disponibilizadas para o sistema de saúde do Brasil, auxiliadas por simplificações nos métodos e normas de vigilância, poderão promover uma melhora geral da efetividade do programa nacional de controle da poliomielite. No último relatório mundial do programa, a possibilidade de alcançar, em caráter global, a interrupção na transmissão da pólio por vírus selvagem, foi proposta para o final do ano de 2003 (27). A principal prioridade do programa deverá ser a reavaliação dos sistemas de vigilância em todas as regiões, pois a segurança do processo de eliminação da transmissão da doença selvagem dependerá de aumentar as exigências de qualidade e efetividade da vigilância epidemiológica. Ao mesmo tempo, devem-se questionar os parâmetros de efetividade utilizados, pois, diante da ausência de casos, novas metodologias de monitoramento podem ser propostas. O grande desafio será continuar a adequar os serviços de monitoramento à realidade de cada continente e país, revertendo diferenças culturais e socioeconômicas em oportunidades de êxito.

\section{REFERÊNCIAS}

1. Pan American Health Organization. Poliomyelitis in the Americas 1969-1984. Bull Pan Am Health Organ 1985;19(4):389-391.

2. Ministério da Saúde, Fundação Nacional de Saúde, Grupo de Trabalho da Pólio. Dossiê do programa de erradicação da transmissão autóctone do poliovírus selvagem no Brasil. Vol. 1. Brasília: Fundação Nacional de Saúde; 1994.

3. De Quadros CA, Hersh BS, Olive JM, Andrus JK, da Silveira CM, Carrasco PA. Eradication of wild poliovirus from the Americas: acute flaccid paralysis surveillance, 1988-1995. J Infect Dis 1997;175(suppl 1):537-542.

4. World Health Organization. The global polio eradication initiative - Strategic plan 20012005. Geneva: WHO, Department of Vaccines \& Biologicals; 2001.

5. World Health Organization. Performance of AFP surveillance and incidence of poliomyelitis 1999-2000. Wkly Epidemiol Rec 2000; 75(37):298-299.

6. De Quadros CA. Global eradication of poliomyelitis and measles: another quiet revolution. Ann Intern Med 1997; 127(2):156-158.

7. Wright PF, Kim-Farley RJ, de Quadros CA, Robertson SE, Scott RM, Ward NA, et al. Strategies for the global eradication of poliomyelitis by the year 2000. New Engl J Med 1991;325(25):1774-1779.

8. World Health Organization. Ata da $53^{\mathrm{a}}$ Assembléia Mundial da Saúde. Informe A53/11, 22 março 2000, ponto 12.9 .

9. Fundação Nacional de Saúde. Guia de vigilância epidemiológica Vol. 1. Brasília: Fundação Nacional de Saúde; 2003.

10. Alter M. The epidemiology of Guillain-Barré syndrome. Ann Neurol 1990;27(suppl):S7S12.
11. Olive JA, Castillo C, Castro RG, de Quadros CA. Epidemiologic study of Guillain-Barré syndrome in children $<15$ years of age in Latin America. J Infect Dis 1997;175(suppl 1):160164.

12. Andrus JK, de Quadros C, Olive JM, Hull HF. Screening of cases of acute flaccid paralysis for poliomyelitis eradication: ways to improve specificity. Bull World Health Organ 1992;70(5):591-596.

13. Pan American Health Organization. Guidelines for epidemiological surveillance - malaria, poliomyelitis. Epidemiol Bull 1999; 20(2):10-13.

14. Instituto Brasileiro de Geografia e Estatística. Disponível em: http://www1.ibge.gov.br/ home/geografia/areaterritorial/principal. shtm\#. Acessado em 26 junho 2003.

15. Waldmann EA. Usos da vigilância e monitorização em saúde pública. Inf Epidemiol SUS 1998;7(3):10-26.

16. Ministério da Saúde, Fundação Nacional de Saúde. Avaliação dos indicadores de qualidade de vigilância epidemiológica e ambiental em saúde. Brasília: Fundação Nacional de Saúde; 2001. Pp. 1-4.

17. World Health Organization, Department of Vaccine \& Biologicals. Polio News 2001; 11. (WHO/POLIO/01.02).

18. Lyra TM, Mendes ACG, Silva Jr JB, Duarte PO, Melo DAM, Albuquerque PC. Sistema de informações hospitalares - fonte complementar na vigilância e monitoramento de doenças imunopreveníveis. Inf Epidemiol Sus 2000; 9(2):87-110.

19. Mendes ACG, Silva Jr JB, Medeiros KR, Lyra TM, Melo Filho DAM, Sá DA. Avaliação do Sistema de Informações Hospitalares (SIH/
SUS) como fonte complementar na vigilância e monitoramento de doenças de notificação compulsória. Inf Epidemiol SUS 2000;9(2):6786.

20. Alexander JP Jr, Gary HE Jr, Pallansch MA. Duration of poliovirus excretion and its implications for acute flaccid paralysis surveillance: a review of the literature. J Infect Dis 1997;175(1):176-182.

21. De Quadros CA, Henderson DA. Disease eradication and control in the Americas. Biologicals 1993;21(4):335-343.

22. PAHO. Polio diagnosis: one or two samples? EPI newsletter 1995;17:51-52.

23. Pinheiro FP, Kew OM, Hatch MH, da Silveira CM, de Quadros CA. Eradication of wild poliovirus from the Americas: wild poliovirus surveillance - laboratory issues. J Infect Dis 1997;175(1):S43-S49.

24. Pan American Health Organization. Report of the Americas. 6th Meeting of the Global Commission for the Certification of Poliomyelitis Eradication. Washington, DC: PAHO; 2001.

25. Birmingham ME, Linkins RW, Hull BP, Hull HF. Poliomyelitis surveillance - the compass for eradication. J Infect Dis 1997;175(suppl 1):S146-S150

26. World Health Organization. Wkly Epidemiol Rec 1999;37(74):305-312.

27. World Health Organization. Global polio eradication initiative - Progress 2002. Geneva: WHO, Department of Vaccines and Biologicals; 2003. (WHO/POLIO/03.02).

Manuscrito recebido em 25 de outubro de 2002. Aceito em versão revisada el 7 de julho de 2003 
ABSTRACT Objectives. To describe and compare indicators of the quality of epidemiological surveillance for acute flaccid paralysis (AFP) before Brazil's certification as a poliomyelitisfree area (1990 through 1994) and after that certification (1995 through 2000).

\section{Indicators of the effectiveness of epidemiological surveillance for acute flaccid paralysis in Brazil from 1990 through 2000}

Methods. The following variables were studied: minimum prevalence (reporting annually at least 1 case of AFP per 100000 individuals younger than 15 years), negative reporting (weekly reporting of the absence of AFP cases by at least $80 \%$ of the reporting units in each region), time of investigation (investigating at least $80 \%$ of the AFP cases within 48 hours of their being reported), and diagnostic investigation (collection of two stool samples for viral culture in the 2 weeks following the onset of paralysis in $80 \%$ or more of the AFP cases). The results were categorized as adequate if they were equal to or higher than the value considered as being effective, or as inadequate if they were below this value. The percentage rate of adequate values was calculated for each of Brazil's 27 federal political divisions (26 states plus the Federal District) in each year, for each of the two periods studied. The final means per period, per federal political division, and per region were also calculated.

Results. For Brazil overall, minimum prevalence decreased from 1.3 per 100000 individuals younger than 15 years in the precertification period to 0.9 per 100000 in the postcertification period, but the difference was not statistically significant $(P>0.08)$. While negative reporting was adequate in both periods (87\% and $84 \%$, respectively), the percentage rate of effectiveness was $6.6 \%$ lower in the postcertification period $(P>0.21)$. The effectiveness in the time required to investigate AFP cases increased from $60.7 \%$ to $71.6 \%(P>0.06)$. The collection of stool samples was inadequate both before and after certification (54\% and $52 \%$, respectively).

Conclusions. Despite the absence of indigenous wild virus poliomyelitis cases in Brazil in the postcertification period, the quality of epidemiological surveillance indicators is still less than desirable. This may delay the detection and investigation of AFP cases and compromise the global poliomyelitis eradication effort. New information strategies that are being made available for the health system in Brazil may improve the effectiveness of the national poliomyelitis control program.

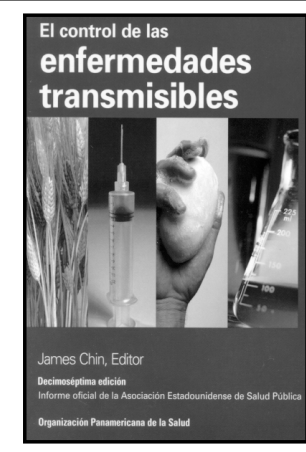

El control de las enfermedades transmisibles, $17^{a}$ ed. ¡Ahora disponible en formato electrónico!

Este libro es una obra de consulta ampliamente conocida sobre las enfermedades transmisibles. Fácil de entender y de usar, contiene información sobre más de trescientas enfermedades. En su decimoséptima edición se han revisado de forma exhaustiva todas las enfermedades comprendidas en la edición anterior y se ha actualizado casi un tercio; se presenta también material adicional sobre las enfermedades víricas por Hendra y Nipah.

Gracias al nuevo formato electrónico, los usuarios podrán comprar la sección específica sobre la enfermedad que les interesa o suscribirse por un año al libro completo en formato electrónico.

Siga los tres pasos siguientes y de inmediato tendrá a su disposición El control de las enfermedades transmisibles en formato electrónico:

* Visite el sitio: http://www.ingentaselect.com

* Seleccione "Pan American Health Organization" en la opción Browse by publishers

* Seleccione el control de las enfermedades transmisibles, 17. a ed. 